Trabajo Original

\title{
Desafío hospitalario: Experiencia de un equipo de ginecología en el manejo integral de pacientes COVID 19
}

\section{A hospital challenge: Experience of a gynecology team in the comprehensive management of patients COVID 19}

Del Pozo Francisca¹, Soto Constanza¹, Véliz Valeria¹, Escobar Paula².

\author{
1 Becadas Ginecología y Obstetricia Hospital Santiago Oriente. Universidad de Chile \\ 2 Jefa unidad Patología mamaria, Hospital Santiago Oriente \\ Centro de procedencia: Hospital Santiago Oriente, Servicio Ginecología y Obstetricia. Universidad de Chile \\ Email del autor: valeria.veliz.valle@gmail.com
}

\section{RESUMEN}

Introducción: La infección por el coronavirus SARS-CoV2 (COVID 19), causal de la pandemia actual, ha significado a nivel mundial la hospitalización simultánea de múltiples pacientes poniendo a prueba la infraestructura hospitalaria y la capacidad de reacción del personal de salud. Una de las estrategias para el manejo es la reconversión de camas y servicios clínicos.

Objetivos: presentar experiencia de un equipo ginecológico en el manejo integral de pacientes no ginecoobstétricas con COVID 19, durante el mes de junio de 2020 en un hospital público de la Región Metropolitana. Métodos: Estudio de corte transversal observacional, descriptivo. Se consideró el total de pacientes adultos hombres y mujeres sin patología gineco-obstétrica con COVID 19 ingresados al puerperio del Hospital Santiago Oriente, obteniéndose datos clínicos y demográficos a través del registro interno de la unidad y del sistema de información de red asistencial.

Resultados: Ingresaron 82 pacientes, 32 mujeres y 50 hombres, promedio de edad 64. El promedio de días de hospitalización fue 5 , con diagnóstico de ingreso principal neumonía viral por COVID-19.

Las comorbilidades frecuentes fueron hipertensión arterial sistémica y diabetes mellitus. La complicación más frecuente fue el tromboembolismo pulmonar agudo.

Hubo una alta cobertura de entrega de información vía telefónica a familiares.

De los 82 ingresos, 54 pacientes egresaron a su domicilio. El resto a otras unidades dentro de la institución, centros de menor complejidad o residencias sanitarias. Una paciente sexo femenino de 75 años fallece a causa de descompensación de patologias de base secundario a neumonia por Staphylococus aereus. En ella, se descarta la infección por COVID 19 dado tres exámenes por reacción de polimerasa en cadena negativos realizado antes y durante su hospitalización.

Conclusiones: Esta experiencia constituyó un desafío para todo el equipo de salud gineco-obstétrico, considerando que nos enfrentamos a otro tipo de pacientes y a una patología nueva. Los resultados médicos son promisorios, la experiencia humana y sentido de trabajo en equipo fue extraordinario.

Palabras claves: Covid - 19, desafío hospitalario, ginecología y obstetricia. 


\section{ABSTRACT}

Introduction: The infection by the SARS-CoV2 coronavirus (COVID 19), the cause of the current pandemic we are experiencing, has meant the simultaneous hospitalization of many patients worldwide, putting the hospital infrastructure and the reaction capacity of health personnel to the test. One of the management strategies is the reconversion of clinical services.

Objectives: present the experience of a gynecological team in the comprehensive management of non-gynecoobstetric patients with COVID 19, during the month of June 2020 in a public hospital in the Metropolitan Region. Methods: descriptive, observational cross-sectional study. The total number of patients admitted to the expuerperium of the Santiago Oriente Hospital was considered, obtaining clinical and demographic data through the unit's internal registry and the healthcare network information system.

Results: 82 patients were admitted, 32 women and 50 men, average age 64 . The average number of days of hospitalization was 5 , with the main admission diagnosis being viral pneumonia due to COVID-19.

Frequent comorbidities were systemic arterial hypertension and diabetes mellitus. The most frequent complication was acute pulmonary thromboembolism.

There was a high coverage of the delivery of information via telephone to relatives. Of the 82 admissions, 54 patients were discharged home and the rest to other units within the institution, less complex centers or health residences. One 75 years old female patient dies from concomitant pathologies, and she wasn't positive for COVID-19.

Conclusions: This experience was a challenge for the entire gynecological-obstetric health team, considering that we are facing other types of patients and a new pathology. The medical results are promising, the human experience and sense of teamwork was extraordinary.

Keywords: Covid-19, Hospital challenge, gynecology and obstetrics.

\section{INTRODUCCIÓN}

El brote actual de la nueva enfermedad por coronavirus (COVID-19) iniciado en diciembre del 2019 ha sido un desafío para todos los sistemas de salud a nivel mundial ${ }^{1}$. El primer caso en Chile es reportado el día 3 de marzo 2020 , alcanzando un total de 279.393 casos y 5688 fallecidos al 30 de Junio de $2020^{2}$.

La Región Metropolitana ha sido el epicentro de este brote, donde se reportan el $78,43 \%$ de los casos totales. Ante este escenario, se ha generado la necesidad de una respuesta sanitaria tanto a nivel ministerial como a nivel loca².

El Hospital Santiago Oriente ubicado en la comuna de Peñalolén atiende a una población de 574.595 usuarios beneficiarios de Fonasa de las nueve comunas del área oriente de Santiago. Para la población de Peñalolén y Macul, además el establecimiento cuenta con servicio de Emergencia Hospitalaria del adulto, con referencia de los Servicios de Atención Primaria de Urgencia (SAPU) de estas comunas $^{3}$. Dentro de las estrategias de manejo de la crisis sanitaria, nace la necesidad de reconversión de la unidad de ginecología - obstetricia puerperio tercer piso, a una unidad de sala básica de medicina interna para pacientes adultos que requieren manejo intrahospitalario, tanto en contexto de infección por COVID 19 u otras patologías de baja complejidad. Esta estrategia se implementa con el objetivo de descongestionar el servcio de urgencia de adultos, unidades de cuidados intermedios y la unidad de medicina interna a cargo de la especialidad.

Desde el 1 de junio del 2020, se crea la unidad con capacidad de 21 camas a cargo del servicio de ginecología y obstetricia del Hospital Santiago Oriente, que recibe pacientes derivados desde los servicios mencionados previamente.

Se destina un equipo de salud conformado por 12 técnicos en enfermería nivel superior (3 por turno), 12 matronas, (3 por turno), una matrona coordinadora de lunes a viernes, 10 médicos becados de ginecología y obstetricia (4 por día de Lunes a Viernes, 2 por turno de fin de semana) y 2 residentes de ginecología y obstetricia (supervisando de Lunes a Viernes). Este 
equipo de salud cambia su quehacer diario habitualmente destinado a usuarias con patología obstétrica y/o ginecológica, para dar respuesta a la contigencia sanitaria.

El objetivo de esta nueva unidad es el manejo y tratamiento principalmente de pacientes con patología respiratoria secundaria a infección por coronavirus. Se considera una unidad de baja complejidad, desde donde se pretende preparar a los pacientes para el alta hospitalaria, o bien derivarlos a otros centros de salud donde puedan completar su hospitalización y/o cuarentena como residencias sanitarias, centro clínico Ñuñoa, Clínica Huechuraba, Instituto Nacional de Geriatría.

Se presenta a continuación una descripción de la experiencia durante el mes de Junio de 2020 de esta unidad.

\section{MATERIALES Y MÉTODOS}

Se realiza estudio de corte transversal observacional, descriptivo.

Considerando el total de pacientes ingresados a la unidad de ex-puerperio desde el 1 de Junio de 2020 hasta el 30 de Junio del mismo año, obteniéndose datos clínicos y demográficos a través del registro interno de la unidad y del sistema de información de red asistencial (SIDRA).

Durante la hospitalización los pacientes recibieron atención integral consistente en kinesiología respiratoria y motora, terapia ocupacional, fonoaudiología. Además, el equipo de salud mental prestó evaluaciones tanto por psicólogo como por psiquiatra cuando fue requerido. Se coordinó apoyo de médicos especialistas en medicina interna para orientaciones clínicas en casos de pacientes con mayor complejidad.

Desde el punto de vista psicosocial, el equipo médico procuró mantener la comunicación permanente de los pacientes con sus familias a través de videollamadas, además se llevó a cabo actualizaciones diarias del estado de salud al tutor a cargo por vía telefónica. Para esto se gestionó la donación de dos equipos móviles, recursos que fueron altamente valorado tanto por los pacientes como por sus familias. Los pacientes y sus familias fueron evaluados por asistente social y utilizando el Score de Riesgo Biosocial fueron categorizados para recibir distintos tipos de ayuda, desde cajas de alimentos, materiales de aseo e incluso apoyo económico 4.

Se diseñaron trípticos informativos de cuidado de pacientes con coronavirus en casa y un instructivo de ejercicios de rehabilitación motora y actividad física entregados a los pacientes con alta a domicilio. Además se implementó un sistema de control telefónico al séptimo día de alta donde se evaluó presencia de signos de alarma, se reforzó la educación de cuidados en domicilio y se derivó el caso a Atención Primaria para su seguimiento y manejo posterior.

Se creó una planilla excel especialmente diseñada y codificada para el posterior análisis estadístico de los datos obtenidos.

\section{RESULTADOS}

Durante el mes de junio de 2020 un total de 82 pacientes ingresaron a la unidad, 32 mujeres (39\%) y 50 hombres $(61 \%)$. La media de edad fue 64 con un intervalo entre (37-91). La mayoría de los pacientes atendidos provenían de una unidad de mayor complejidad, el promedio de días hospitalizados previo a su ingreso fue de 12, con un intervalo de (1$60)$. El promedio de días de hospitalización en nuestra unidad fue de 5 . El diagnóstico de ingreso para 77 pacientes fue neumonía viral por COVID 19. Las principales comorbilidades fueron hipertensión arterial sistémica (56\%), diabetes mellitus (40\%) y Obesidad (20\%).

La complicación más frecuente durante la hospitalización en la unidad fue el tromboembolismo pulmonar agudo, que afectó a 10 pacientes (12\%), de los cuales 7 fueron hombres (70\%) y 3 mujeres (30\%). La segunda complicación más frecuente fue la injuria renal aguda que afectó a 5 pacientes (6\%), de los cuales 4 fueron hombres(80\%) y 1 mujer (20\%),

Una paciente sexo femenino, de 75 años con antecedente de insuficiencia cardiaca, hipertensión arterial y trombosis venosa profunda/tromboembolismo pulmonar con filtro de vena cava, fallece a causa de descompensación de patologias de base secundario a neumonia por Staphylococus aereus. En ella, a pesar de su negativa evolución, se descarta la infección por COVID 19 dado tres exámenes por reacción de polimerasa en cadena 
para SARS-CoV2 negativos realizado antes y durante su hospitalización.

En relación al alta de la unidad; 54 pacientes (66\%) fueron egresados a su domicilio, 8 (10\%) requirieron traslado a unidades de mayor complejidad dentro de la misma institución, 2 (2\%) fueron derivados a su centro de origen (institucionalizados en centros de adultos mayores), 1 (1\%) a centro de rehabilitación, 7 (9\%) a residencia sanitaria, 7 (9\%) a centros de menor complejidad (Centro clínico Ñuñoa) y $2(2 \%)$ permanecen hospitalizados a la fecha.

De los pacientes dados de alta a domicilio, se consideró necesario realizar control telefónico en 15 de ellos. En este control, 14 evolucionaron favorablemente con indicación de continuar control ambulatorio en sus respectivos centros de salud familiar o en nivel secundario si así lo que requerían. Una paciente requirió rehospitalización en el servicio de medicina.

\section{CONCLUSIONES}

En nuestra unidad hubo más ingresos de pacientes de sexo masculino que femenino, los cuales además presentaron más complicaciones, similar a lo que se ha reportado en la actualidad.

Esta experiencia ha sido un desafío para todo el equipo de salud, por la necesidad de reconversión de infraestructura y el cambio diametral de las actividades clínicas diarias del equipo de ginecología y obstetricia, considerando que nos enfrentamos a otro tipo de pacientes y a una patología nueva en un contexto de pandemia. Requirió capacitación y adquisición de nuevas competencias en un tiempo acotado a medida que se daba respuesta a las necesidades sanitarias inmediatas.
Cabe destacar que estas nuevas tareas fueron asumidas por el personal de salud con actitud de entrega, espíritu positivo y profesionalismo.

Se implementó una atención integral, poniendo énfasis tanto en lo clínico como en lo humano, mostrando preocupación por la salud mental enfermo y la soledad que viven durante la hospitalización actual con prohibición de visitas, haciendo partícipe a la familia desde la llegada de los usuarios a la unidad hasta el control telefónico en domicilio.

Los resultados médicos fueron promisorios, considerando el manejo de patologías de medicina interna por una unidad de ginecología y obstetricia. La experiencia humana y sentido de trabajo en equipo fue única.

\section{REFERENCIAS}

1. ORGANIZACIÓN MUNDIAL DE LA SALUD. (2020). 30/06/20, de WHO Sitio web: https://www.who.int/es/news-room/detail/29-062020-covidtimeline

2. Ministerio de salud de Chile. (2020). Reporte de casos COVID-19, de MINSAL Sitio web:https://www.minsal.cl/nuevo-coronavirus2019-ncov/casos-confirmados-en-chile-covid-19/

3. Hospital Santiago Oriente Luis Tisné Brousse.(2020), Hospital Santiago Oriente.

4. Sitio web: http://www.hsoriente.cl/001.php

5. Score de Riesgo Biosocial. Ordinario 2403, Subsecretaria de Redes Asistenciales , Agosto 2016. 
ANEXOS

ANEXO 1. tríptico informativo cuidados paciente covid-19 en domicilio

TIPS PARA LA CASA

$\Rightarrow \quad V E N T$ I L A R FRECUENTEMENTE

$\Rightarrow \quad$ LAVADO Y CAMBIO DE ROPA FRECUENTE.

$\Rightarrow$ ASEO DIARIO DE SUPERFICIES DE CON TACTO MESONES, MANILLAS, B A N OS CON SOLUCION CLORADA.

$\Rightarrow$ PREPARACION $S O L U C I O N$ CLORADA:

$\Rightarrow \quad 4$ CUCHARADITAS (TE) DE CLORO EN UN LITRO DE AGUA EN BOTELLA LIMPIA Y TAPADA E IDEALMENTE CON ROCIADOR (LO QUE SOBRA DEBE SER QUE SOBRA DEBE SER E L I M I N A D
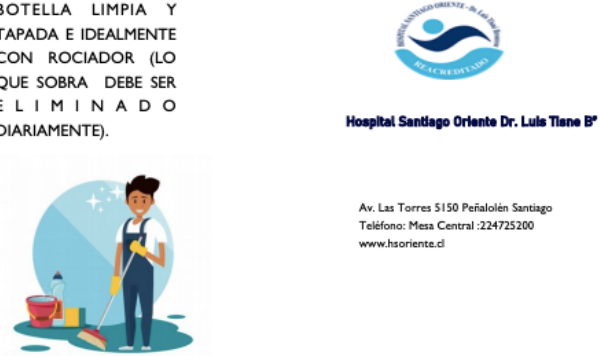

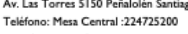

HOSPITAL SANTIAGO ORIENTE

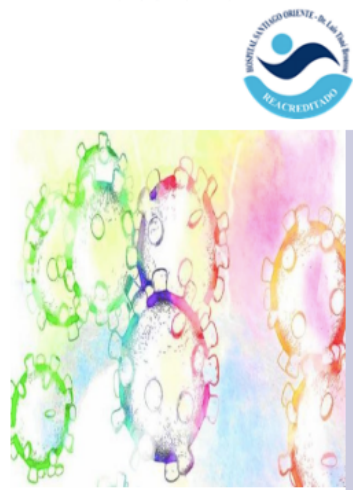

CUIDADOS DEL ENFERMO CON

ENFERMEDAD

RESPIRATORIA POR

CORONAVIRUS EN

DOMICILIO

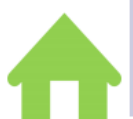

CUIDADOS DEL ENFERMO CON ENFERMEDAD RESPIRATORIA POR CORONAVIRUS EN DOMICILIO

1. DORMIR EN CAMA SEPARADA

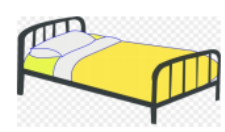

2. SI ES POSIBLE UTILIZAR BAÑO DIFERENTE AL RESTO DE LOS HABITANTES DE LA CASA O UNA VEZ UTILIZADO POR EL PACIENTE SE DEBE LIMPIAR CON SOLUCION CLORADA.

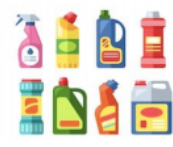

3. SEPARAR UTENSILIOS COMO CUBIERTOS, VASOS TOALLAS.

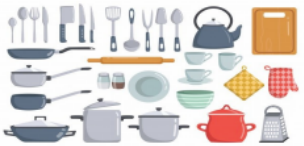

4. MANTENER DISTANCIA DE UN METRO CON EL ENFERMO Y LAVARSE LAS MANOS CON AGUA $Y$ JABON ANTES $Y$ DESPUES DE ATENDERLO

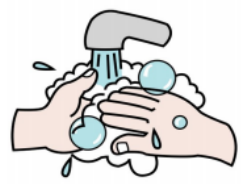

5. SEGUIR LAS

INDICACIONES QUE

ENTREGO EL MEDICO Y PERMANECER EN

CUARENTENA JUNTO A

LOS HABITANTES DE LA

SIGNOS DE ALARMA

- sentir ahogo O DIFICULTAD PARA RESPIRAR,

- en este caso acuda NUEVAMENTE AL

SERVICIO DE URGENCIA MAS CERCANO. 
ANEXO 2. recomendaciones ejercicio respiratorio y físico en domicilio.

\section{RECOMENDACIONES de ejercicio respiratorio y físico para personas afectadas por COVID-19 \\ EJERCICIOS RESPIRATORIOS PARA RESTABLECER EL PATRÓN VENTILATORIO Y EL VOLUMEN PULMONAR: \\ érs}

\begin{tabular}{|c|c|c|c|}
\hline $\begin{array}{l}\text { Respiración labios fruncidos: } \\
\text { Realizar este ejercicio puede ayudar a reducir la sensación de disnea } \\
\text { o dificultad respiratoria. } \\
\text { Inspirar lentamente por la nariz. } \\
\text { Aguantar el aire } 2-3 \text { segundos, si se puede. } \\
\text { Soplar lentamente por la boca formando una U con los labios. }\end{array}$ & & & \\
\hline $\begin{array}{l}\text { Respiración abdominal o diafragmática } \\
\text { Acostado con piernas semiflectadas (se puede poner un cojín debajo) } \\
\text { o sentado en una silla. Manos en el abdomen para notar como se } \\
\text { levanta al tomar aire (inspirar) y se hunde al sacar el aire (espirar). } \\
\text { Tomar aire por la nariz (el máximo que se pueda) y sacarlo } \\
\text { lentamente por la boca con los labios fruncidos. Realizar por la } \\
\text { mañana y por la tarde, 10-15 respiraciones. }\end{array}$ & & & $\pi 14$ \\
\hline $\begin{array}{l}\text { Respiración costal (ejercicios de expansión torácica): } \\
\text { Acostado con piernas estiradas o sentado en una silla. Manos en el } \\
\text { tórax para notar como se infla al tomar aire (inspirar) y se desinfla al } \\
\text { sacar el aire (espirar). Tomaraire por la nariz (el máximo que se } \\
\text { pueda) y sacarlo lentamente por la boca con los labios fruncidos. } \\
\text { Realizar por la mañana y por la tarde, 10-15 respiraciones. }\end{array}$ & 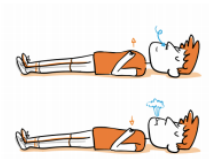 & गाग & (1T) \\
\hline
\end{tabular}

EJERCICIOS FíSICOS PARA LA READAPTACIÓN A LAS ACTIVIDADES DE LA VIDA DIARIA

\begin{tabular}{|l|l|}
\hline $\begin{array}{l}\text { Realizar ejercicio físico a diario (según sintomatología en aquellos sujetos que estén afectados por el covid- } \\
\text { 19). No se recomienda realizar esfuerzo si se está con fiebre o mucha sintomatología respiratoria (mucha } \\
\text { sensación de ahogo o dificultad al respirar). }\end{array}$ \\
\hline $\begin{array}{l}\text { Evitar periodos largos sentado o inmóvil: Cada hora se recomienda realizar } 1 \text { ejercicio aeróbico y } 2 \text { ejercicios } \\
\text { de fuerza resistencia (uno de brazos y uno de piernas). }\end{array}$ \\
\hline $\begin{array}{l}\text { Controlar la respiración durante los ejercicios, inspirando durante la contracción muscular y espirando } \\
\text { durante la relajación (se puede usar los labios fruncidos en la espiración). }\end{array}$ \\
\hline Beber líquido frecuentemente durante el día.
\end{tabular}

\section{EJERCICIOS DE ENTRENAMIENTO DE FUERZA-RESISTENCIA:}

Frecuencia: a diario.

Intensidad: realizar tantas repeticiones del movimiento hasta llegar a una fatiga del grupo muscular utilizado (o sensación de dificultad respiratoria) Dosis: se realizarán 2-3 series (según tolerancia), y se descansará entre

30 " y 1 ' (según capacidad de recuperación) entre series de ejercicio. Tipo: Se recomiendan ejercicios que sigan movimientos funcionales,. Algunos ejemplos son:

- Para extremidad inferior: sentarse- levantarse de una silla, subir-bajar escalones, agacharse doblando rodillas (sentadillas) y volverse a levantar.

- Para extremidad inferior: subir-bajar pesos de una altura parecida a un armario, coger una bolsa/botella de agua/peso con una mano y levantarla flexionando el codo siguiendo el lateral de la pierna con la mano, delante de una pared hacer

flexiones de brazos (si se puede, realizar en el suelo)

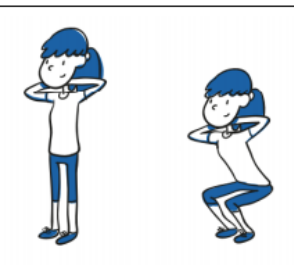




\section{EJERCICIOS DE ENTRENAMIENTO DE RESISTENCIA AERÓBICA}

Frecuencia: a diario.

Intensidad: un ejercicio de intensidad moderada corresponderá en la sensación de dificultad respiratoria (o fatiga) de 5-6, mientras que un ejercicio intenso corresponderá a 7-8.

Tiempo: mínimo 10 minutos, ideal 30 minutos.

Tipo: se recomienda ejercicios que impliquen grandes grupos musculares. Y se realizará trabajo por series. Siguiendo esta pauta: 30" o 1' de trabajo (sensación en escala de Borg moderada (5-6) para sujetos con una forma física más limitada, sensación intensa (7-8) para aquellos que tengan mejor forma física)

con 1' de descanso (sensación en escala de Borg debería bajar entre 0 y 3).

Ejercicios propuestos: subir y bajar escaleras (o un escalón), caminar por la casa (aunque el espacio sea limitado), saltar o correr en el lugar.

\section{ESCALA DE BORG:}

La escala de Borg modificada sirve para valorar la percepción subjetiva de esfuerzo y puntúa desde 0 (corresponde a nada de fatiga o de dificultad respiratoria) y aumenta hasta 10 (que corresponde a la máxima fatiga o dificultad respiratoria). Es una buena herramienta para el control de la intensidad del trabajo o ejercicio realizado.
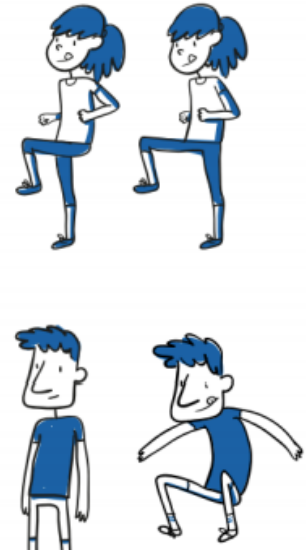

\begin{tabular}{|rl|}
\hline 10 & Máximo \\
9 & Muy, muy fuerte \\
8 & \\
7 & Muy fuerte \\
\hline 6 & \\
5 & Fuerte \\
4 & Un poco fuerte \\
\hline 3 & Regular \\
2 & Poco \\
1 & Muy poco \\
0 & Nada \\
\end{tabular}

